\title{
Pre-treatment lactate dehydrogenase levels as predictor of efficacy of first-line bevacizumab-based therapy in metastatic colorectal cancer patients
}

\section{Scartozzi", , R Giampieri', E Maccaroni ${ }^{2}$, M Del Prete ${ }^{2}$, L Faloppi ${ }^{2}$, M Bianconi ${ }^{2}$, E Galizia ${ }^{3}$, C Loretelli', L Belvederesi', A Bittoni' ${ }^{2}$ and S Cascinu'}

'Clinica di Oncologia Medica, AO Ospedali Riuniti, Università Politecnica delle Marche, Via Conca, Ancona, Italy; ${ }^{2}$ Scuola di Specializzazione in Oncologia Medica, Università Politecnica delle Marche, Ancona, Italy; ${ }^{3}$ Oncologia Medica, Ospedale Profili, Fabriano, Italy

BACKGROUND: Lactate dehydrogenase (LDH) represents a predictive factor in colorectal cancer patients treated with the angiogenesis inhibitor PTK/ZK. We explored the role of pre-treatment LDH serum levels in colorectal cancer patients receiving first-line bevacizumab.

METHODS: Metastatic colorectal cancer treated with first-line bevacizumab was eligible. A control group including all consecutive patients treated with chemotherapy alone was also considered. Pre-treatment LDH serum levels were collected for all cases. RESULTS: Median progression-free survival (PFS) in the control group for patients with high and low LDH levels was 4.2 and 8 months, respectively $(P=0.0003)$. Median overall survival $(O S)$ was 19.6 and 34.9 months for patients with high and low LDH levels, respectively $(P=0.0014)$. In the bevacizumab group, partial responses were seen in $14(58 \%)$ high-LDH and $8(14 \%)$ low-LDH patients $(P=0.0243)$, respectively, median PFS was 7.3 and 8.5 months, respectively $(P=0.2)$, and median OS was 22 and 26.6 months, respectively $(P=0.7)$.

CONCLUSION: High LDH levels correlated with worse prognosis. Bevacizumab seemed capable of improving clinical outcome in this specific group of patients who usually present with an adverse natural history. The improved response rate also suggests a role for $\mathrm{LDH}$ as a predictive marker.

British Journal of Cancer (2012) 106, 799-804. doi:10.1038/bjc.2012.17 www.bjcancer.com

Published online 7 February 2012

(C) 2012 Cancer Research UK

Keywords: bevacizumab; colorectal cancer; LDH; predictive factors

The VEGF-driven tumour pathway has been demonstrated to represent a novel therapeutic target for an innovative class of antineoplastic agents. Among these antiangiogenetic-targeted treatment modalities, the anti-VEGF monoclonal antibody bevacizumab has become a new standard of care for first-line treatment of metastatic colorectal cancer (Douillard et al, 2000; Hurwitz et al, 2004; Koehne et al, 2005).

The expanding role of anti-VEGF treatment for metastatic colorectal tumour patients, along with the growing number of cases potentially requiring such a treatment approach, made the need for a reliable identification of responding tumours increasingly crucial (Folkman, 1971).

However, if on the one hand clinical reports with the use of bevacizumab have shown promising results, on the other hand in these trials no predictive markers of response or resistance were identified.

The biological link between hypoxia, lactate dehydrogenase (LDH) levels and the tumour-driven angiogenesis pathway through the abnormal activation of the hypoxia-inducible factor $1-\alpha($ HIF $1-\alpha)$ is well established. Hypoxia-inducible factor $1-\alpha$ is an

*Correspondence: Dr M Scartozzi; E-mail: marioscartozzi@libero.it Received 21 October 2011; revised II January 2012; accepted II January 2012; published online 7 February 2012 important transcription factor that upregulates a series of genes involved in glycolytic metabolism, angiogenesis, cell survival and erythropoiesis. Among the others, HIF1- $\alpha$ also regulates transcription of several glycolytic enzymes, such as LDH (Maxwell et al, 2001). More specifically, it has been suggested that HIF1- $\alpha$ overexpression was linked to the LDH-5 isoform activity (Koukourakis et al, 2003).

In preclinical models, upregulation of LDH has been suggested to ensure both an efficient anaerobic/glycolytic metabolism and a reduced dependence on oxygen under hypoxic conditions in tumour cells.

As LDH and pro-angiogenesis factors are regulated by the same HIF1- $\alpha$-driven molecular pathway, high LDH levels are concomitantly present along with abnormal activation of the VEGF pathway (Harris, 2002).

According to this biological assumption, Azuma et al (2007) demonstrated that high LDH serum levels were associated with tumour overexpression of VEGFA and VEGFR-1. As a clinical consequence, it has been speculated that LDH levels may represent an indirect indicator of activated tumour angiogenesis and ultimately of worse prognosis (Tas et al, 2001a, b).

In colorectal cancer, LDH-5 overexpression has been demonstrated to significantly correlate with an increased risk of metastases, and high LDH serum levels have been implicated in determining a worse prognosis (Koukourakis et al, 2005; 
Wu et al, 2010). This finding has been confirmed in other tumour types as well (Brizel et al, 2001; Yuce et al, 2001).

The role of $\mathrm{LDH}$ in patients receiving antiangiogenic therapy is more controversial. Available evidences in this setting are limited to those deriving from the CONFIRM-1 and -2 trials. In these studies, PTK/ZK (vatalanib), an oral inhibitor of VEGF receptors, was used in combination with chemotherapy (FOLFOX) for, respectively, first- and second-line therapy of advanced colorectal cancer (Hecht et al, 2011; Van Cutsem et al, 2011). Both trials did not meet the primary end point. However, in an exploratory post hoc analysis, median progression-free survival (PFS) improved with the use of $\mathrm{PTK} / \mathrm{ZK}$ in patients with high LDH serum levels, thus suggesting that $\mathrm{LDH}$ might be a predictive marker for antiangiogenic treatment.

Recently, Koukourakis et al (2011) also demonstrated that serum LDH and tissue LDH-5 are complementary features that may help characterising the activity of LDH in colorectal cancer.

On the other hand, data in colorectal cancer patients receiving first-line bevacizumab are lacking and could be relevant for treatment strategy and therapeutic decision in clinical practice. The aim of our study was to explore a possible link between pre-treatment LDH levels and clinical outcome in advanced colorectal cancer patients treated with first-line chemotherapy and bevacizumab.

\section{PATIENTS AND METHODS}

\section{Patient selection}

All patients with histologically proven metastatic colorectal cancer consecutively treated with a first-line chemotherapy doublet and bevacizumab at our Institution were eligible for our analysis. A historical control group was also created, including all consecutive histologically proven metastatic colorectal cancer patients treated at our Institution with a chemotherapy doublet before the introduction of bevacizumab in clinical practice. Pretreatment LDH serum levels were collected for all patients.

The following first-line chemotherapy doublets were used: modified FOLFIRI (irinotecan $180 \mathrm{mg} \mathrm{m}^{-2} \mathrm{~d} 1$, 5FU bolus $400 \mathrm{mg} \mathrm{m}^{-2}$ $\mathrm{d} 1,5 \mathrm{FU} 2400 \mathrm{mg} \mathrm{m}^{-2}$ continuous infusion for $46 \mathrm{~h}$ every 2 weeks) or FOLFOX-6 (oxaliplatin $85 \mathrm{mg} \mathrm{m}^{-2} \mathrm{~d} 1$, 5FU bolus $400 \mathrm{mg} \mathrm{m}^{-2} \mathrm{~d} 1$, 5FU $2400 \mathrm{mg} \mathrm{m}^{-2}$ continuous infusion for $46 \mathrm{~h}$, every 2 weeks) or XELOX (oxaliplatin $130 \mathrm{mg} \mathrm{m}^{-2} \mathrm{~d} 1$, capecitabine $2000 \mathrm{mg} \mathrm{m}^{-2} \mathrm{~d} 1$ to 14 every 3 weeks) either in combination with bevacizumab $\left(5 \mathrm{mg} \mathrm{kg}^{-1}\right.$ every 2 weeks or $7.5 \mathrm{mg} \mathrm{kg}^{-1}$ every 3 weeks) or without bevacizumab.

Tumour response was evaluated every 8 weeks by clinicians' assessment and according to the Response Evaluation Criteria in Solid Tumors (RECIST).

\section{Statistical analysis}

Statistical analysis was performed with the MedCalc package (MedCalc v.9.4.2.0, MedCalc Software bvba, Mariakerke, Belgium). Receiver operating characteristics (ROC) curve analysis was performed to determine a cutoff value for pre-treatment LDH levels.

The association between categorical variables was analysed by $\chi^{2}$-test. Survival distribution was estimated by the Kaplan-Meier method. Significant differences in probability of relapsing between the strata were evaluated by log-rank test. Cox multiple regression analysis was used to assess the role of variables that resulted to be significant at univariate analysis.

Tested variables included gender (male $v s$ female), age ( $<65 v s$ $\geqslant 65$ years), grade of tumour differentiation (well $v s$ moderately differentiated and undifferentiated), Eastern Cooperative Oncology Group Performance Status Scale (ECOG PS) $(<2 v s \geqslant 2)$ and LDH serum level $\left(\leqslant 588 v s>588 \mathrm{mg} \mathrm{dl}^{-1}\right)$.

The heterogeneity of the effect of LDH levels between bevacizumab and historical control group was explored by using a statistical test for interaction, applied through a Cox model for PFS and overall survival (OS).

A significant level of 0.05 was chosen to assess the statistical significance.

For statistical analysis, OS and PFS were defined, respectively, as the interval between the start of chemotherapy to death or last followup visit, and as the interval between the start of chemotherapy to clinical progression or death, or last follow-up visit if not progressed.

\section{RESULTS}

Globally, 220 patients with advanced colorectal cancer receiving first-line chemotherapy were available for our analysis. In all, 82 patients were treated with a chemotherapy doublet (either oxaliplatin or irinotecan in combination with fluoropyrimidines) in combination with bevacizumab (bevacizumab group; accrual interval 2005-2011), whereas the remaining 138 patients received chemotherapy (either oxaliplatin or irinotecan in combination with fluoropyrimidines) alone (historical control group; accrual interval 1999-2005). The two groups of patients were comparable for all major clinical characteristics such as age at diagnosis, sex, metachronous $v s$ synchronous metastatic involvement, previous adjuvant chemotherapy, number of metastatic sites and proportion receiving second-line treatment (Table 1 ). The cutoff point with the highest sensitivity and specificity for estimating pre-treatment LDH serum levels as a function of treatment of clinical activity was set at $\leqslant 588 \mathrm{mg} \mathrm{dl}^{-1}$ after ROC curve analysis (Figure 1). Conse-

Table I Patients characteristics

\begin{tabular}{lccccc}
\hline & Median age & Males (\%) & $\begin{array}{c}\text { Metachronous } \\
\text { disease (\%) }\end{array}$ & $\begin{array}{c}\text { Prior adjuvant } \\
\text { chemotherapy (\%) }\end{array}$ & $\begin{array}{c}\text { Metastatic } \\
\text { involvement } \\
\text { (A-B-C-D) }\end{array}$ \\
\hline (range) & N & 2nd line (\%)
\end{tabular}

Abbreviations: $\mathrm{A}=$ liver involvement only; $\mathrm{B}=$ lung involvement only; $\mathrm{C}=$ liver+lung involvement; $\mathrm{D}=$ other metastatic sites. 
quently, patients showing a pre-treatment LDH serum level $\geqslant 588 \mathrm{mg} \mathrm{dl}^{-1}$ were classified as high-LDH patients, in contrast to patients with pre-treatment LDH serum level lower than $588 \mathrm{mg}$ $\mathrm{dl}^{-1}$ (low-LDH patients). Globally, 44 patients (20\%) showed high pre-treatment LDH levels.

\section{Role of pre-treatment LDH levels in the historical control group}

In all, 138 patients were available for analysis in this group. Of these, 49 patients showed a partial response (35\%), 1 patient obtained a complete remission (1\%), 32 patients achieved a stable disease $(23 \%)$ and 40 patients (29\%) progressed during chemotherapy. The remaining 16 patients $(12 \%)$ were not assessable for response. In the chemotherapy-alone group, 20 patients $(15 \%)$ had high pre-treatment LDH levels. The remaining 118 patients (85\%) presented with low pre-treatment LDH levels. The two subgroups of patients were comparable for all major clinical characteristics (Table 1).

In high- and low-LDH patients, we observed a partial response in $4(20 \%)$ and $46(39 \%)$ patients, respectively $(P=0.1671)$, whereas progressive disease was observed in $14(70 \%)$ and 26

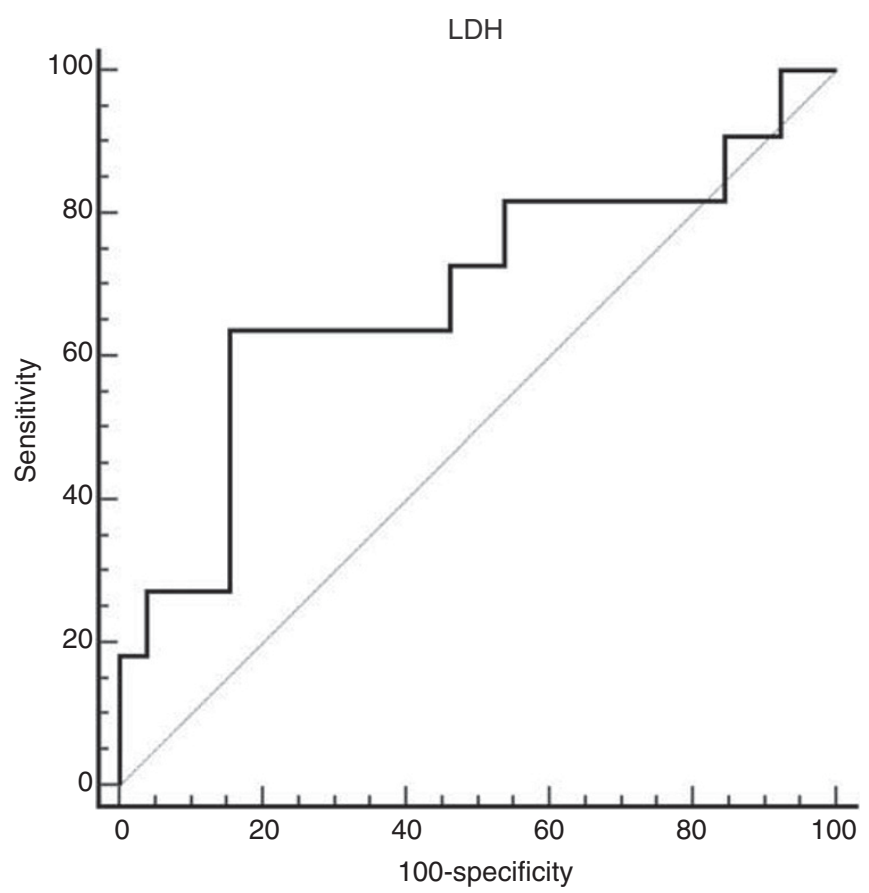

Figure I Receiver operating characteristics analysis based on pretreatment LDH serum levels results with $O S$ as end point. In this model, sensitivity was $63.64 \%$ (95\% Cl: $40.7-82.8$ ) and specificity was $84.62 \%$ (95\% Cl: 71.9-93.I). Area under the curve was 0.689, $P=0.0077$.
(22\%) patients with, respectively, high and low pre-treatment LDHlevels $(P<0.0001$; Table 2$)$.

Median PFS and OS for patients in the historical control group were, respectively, 7.2 and 29.7 months. In this group, pre-treatment LDH levels were statistically related to both worse median PFS and OS. In particular, patients with high LDH levels achieved a median PFS of 4.2 months, whereas median PFS was 8 months for patients with low pre-treatment LDH levels $(P=0.0003$; HR: 0.2973 ; 95\% CI: 0.0318-0.3543; Figure 2 and Table 2). Median OS was 19.6 and 34.9 months for patients with high and low pre-treatment LDH levels, respectively ( $P=0.0014$; HR: 0.2484 ; $95 \%$ CI: $0.0188-0.3884$; Figure 3 and Table 2). Among the other tested clinical parameters, median OS improved in male patients (median OS in male $v s$ female patients: 33.5 vs 17.8 months, respectively, $P=0.007$ ) and in patients with a well-differentiated tumour (median OS in patients with welldifferentiated $v s$ moderately differentiated and undifferentiated tumours: not reached $v s 25.5$ months, respectively, $P=0.007)$. At multivariate analysis, only LDH serum level maintained an independent prognostic value.

\section{Role of pre-treatment LDH levels in the bevacizumab group}

In all, 82 patients were available for analysis in this group. Of these, 22 patients showed a partial response (27\%), 40 patients obtained a stable disease (49\%), whereas 12 patients (15\%) progressed during treatment. In the remaining eight patients (9\%), response was not assessable. No complete remissions were observed. In the bevacizumab group, 24 patients $(29 \%)$ had high pre-treatment LDH levels. The remaining 58 patients $(71 \%)$ presented with low pre-treatment LDH levels. The two subgroups of patients were comparable for all major clinical characteristics (Table 1). In highand low-LDH patients, we observed a partial response in $14(58 \%)$ and $8(14 \%)$ patients, respectively $(P=0.0243)$, whereas progressive disease was observed in $2(8 \%)$ and $10(17 \%)$ patients with, respectively, high and low pre-treatment $\mathrm{LDH}$ levels, respectively $(P=0.48$; Table 2). Median PFS was 8.5 and 7.3 months, respectively, for high- and low-LDH patients $(P=0.2$; HR: 0.6360 ; 95\% CI: $0.2528-1.4185$ ) (Figure 2 and Table 2). Median OS survival was 26.6 and 22 months, respectively, for patients showing high or low pre-treatment LDH levels $(P=0.7$; HR: 0.8480 ; 95\% CI: 0.2307-2.9563) (Figure 3 and Table 2). All the other tested variables (age, gender, ECOG PS and tumour grade) failed to show a significant correlation with survival parameters.

\section{Combined results}

We compared results from the bevacizumab and control groups according to pre-treatment LDH levels. No statistically significant differences were noticed for median OS. In the high-LDH group, response rate was higher in patients treated with bevacizumab ( $58 \%$ vs $20 \%, P=0.01$ ). Accordingly, median PFS in the high-LDH

Table 2 Response rate according to pre-treatment LDH levels in the bevacizumab group and in the control group

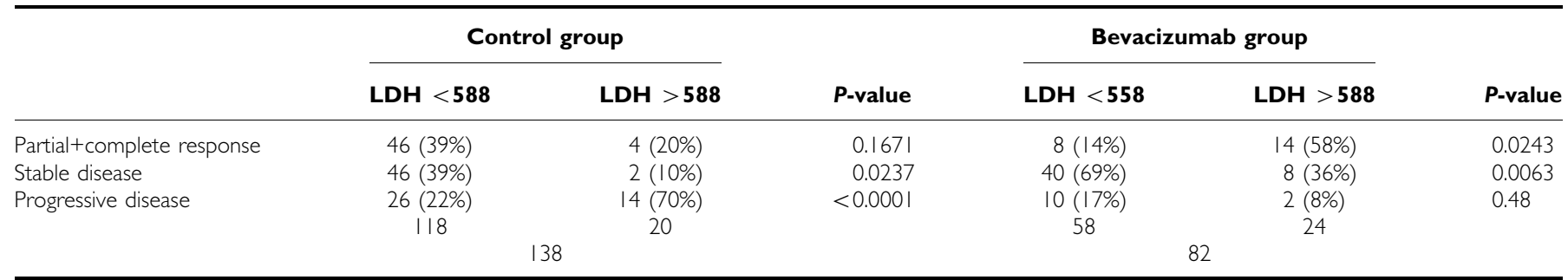

Abbreviation: $\mathrm{LDH}=$ lactate dehydrogenase. 

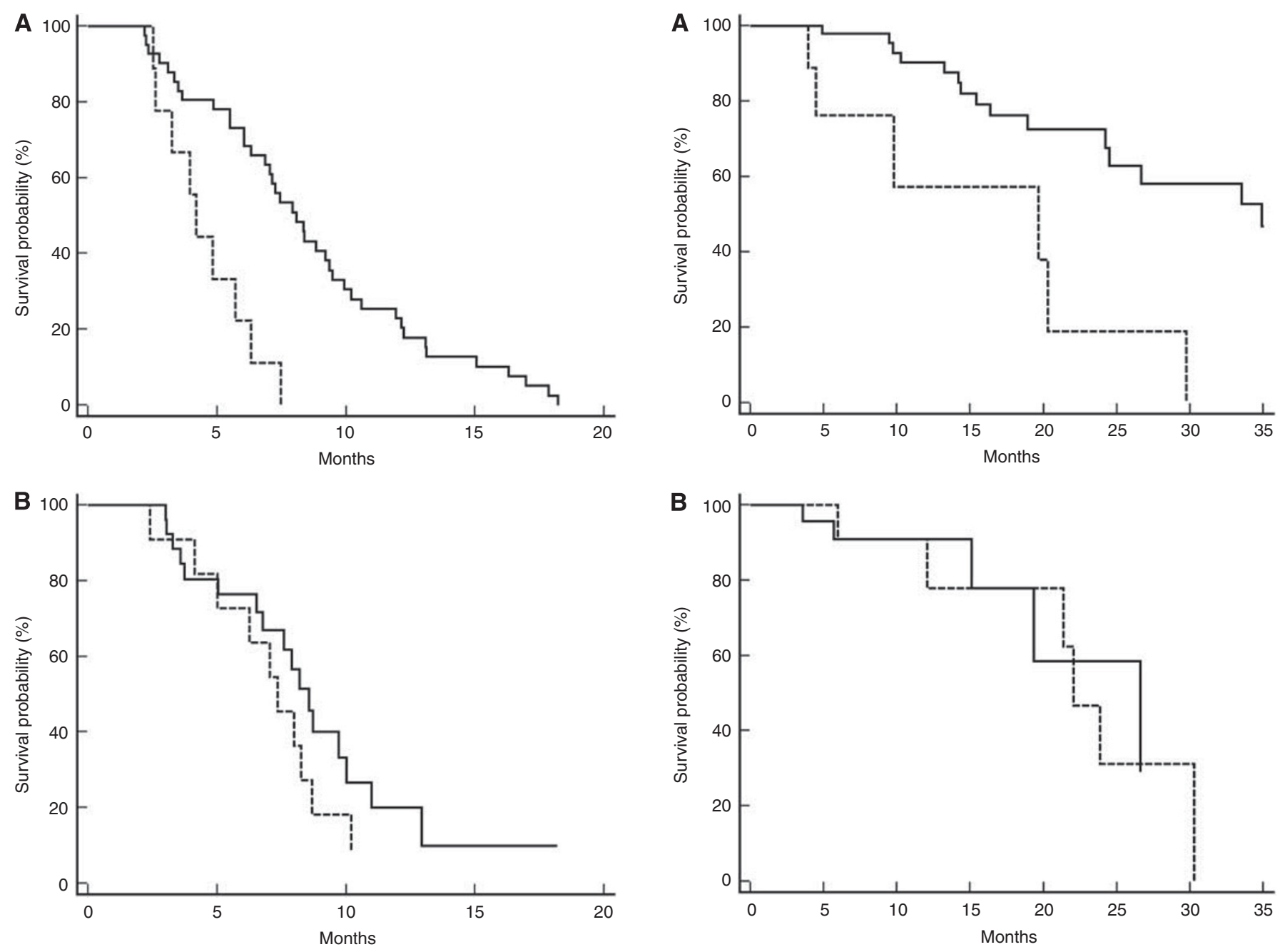

Figure 2 (A) Kaplan-Meier curves for median PFS of colorectal cancer patients in the historical control group showing high pre-treatment LDH serum levels ( . . . . - ) and low pre-treatment serum levels $(-\vec{C})$ (4.2 vs 8 months, $P=0.0003$ ). (B) Kaplan-Meier curves for median PFS of colorectal cancer patients in the bevacizumab group showing high pre-treatment LDH serum levels (- - . - - -) and low pre-treatment serum levels $(-)$ ( 8.5 vs 7.3 months, $P=0.2)$.

group was in favour of patients treated with bevacizumab $(P=0.006$; HR: 3.6257; 95\% CI: $1.5776-16.0715$; Figure 4$)$. The interaction test between LDH levels and treatment effect, in the bevacizumab group and historical control group, suggested that the relation of LDH levels with improved outcome was significantly associated with the effect of bevacizumab in terms of PFS $(P=0.02)$, but not in terms of OS $(P=0.07)$.

\section{DISCUSSION}

The role of $\mathrm{LDH}$ as a marker of clinical outcome in advanced colorectal cancer patients has been suggested in different clinical series. Previous reports in colorectal cancer patients indicated, in fact, that LDH upregulation was associated with an increased risk of both nodal and distant metastases (Tas et al, 2001a), and that high LDH serum levels could predict a decreased median OS and have a prognostic impact (De Gramont et al, 2000; Tas et al, 2001b; Tournigand et al, 2004, 2011). This analysis suggested that pretreatment LDH levels could be considered a relevant factor for risk evaluation in colorectal cancer patients receiving chemotherapy.

Figure 3 (A) Kaplan-Meier curves for median OS of colorectal cancer patients in the historical control group showing high pre-treatment LDH serum levels (- . - . - - ) and low pre-treatment serum levels ( (19.6 vs 34.9 months, $P=0.00$ |4). (B) Kaplan-Meier curves for median OS of colorectal cancer patients in the bevacizumab group showing high pre-treatment LDH serum levels (- - - - - ) and low pre-treatment serum levels $(-)$ (26.6 vs 22 months, $P=0.7)$.

Besides representing a possible indicator of prognosis, LDH may also have a role in patient stratification for clinical trials investigating first-line therapy in colorectal tumours. However, although a biological relationship between LDH levels, tumour hypoxia and angiogenesis has been indicated in pre-clinical and clinical studies, the role of pre-treatment LDH levels in patients receiving anti-VEGF therapy remains substantially unexplored. The only available data about the role of $\mathrm{LDH}$ levels and an antiangiogenic treatment are those deriving from the recently published trials investigating PTK/ZK (vatalanib), an oral inhibitor of VEGF receptors, in first- and second-line therapy of advanced colorectal cancer. Both trials did not reach their primary end point, but in an exploratory post hoc analysis median PFS improved with the use of PTK/ZK in patients with high serum LDH level. No meaningful effect was seen on either response rate or OS. The strong association between LDH-5 expression and the activated VEGF pathway demonstrated in a study by Koukourakis et al (2006) may represent a biological rationale to explain anti-VEGF activity in the presence of high LDH levels.

Interestingly, our analysis suggested that metastatic colorectal cancer patients treated with bevacizumab and showing high 


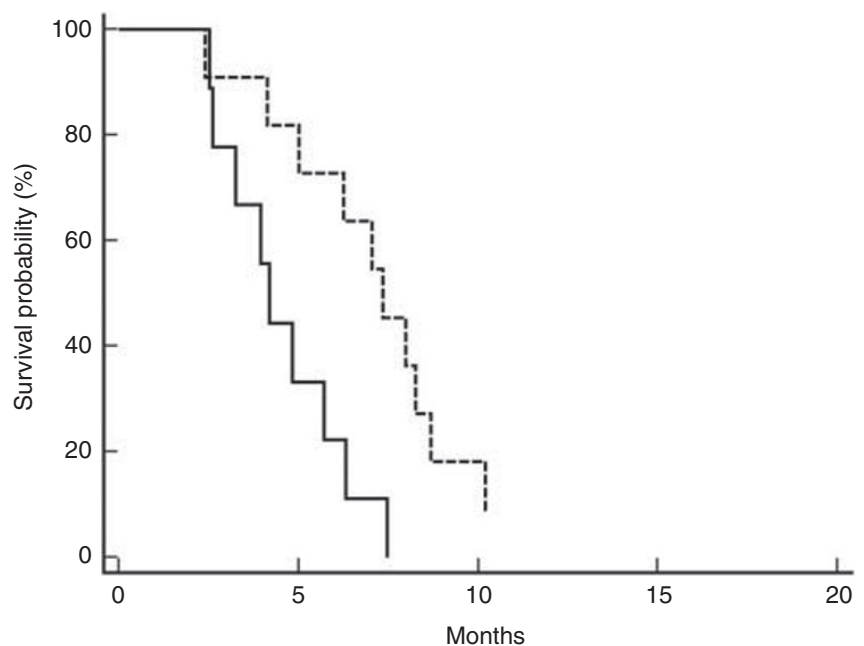

Figure 4 Kaplan-Meier curves for median PFS of colorectal cancer patients showing high pre-treatment LDH serum levels. Bevacizumab group (- - - - -) and historical control group ($P=0.006)$.

pre-treatment LDH levels experienced an improved probability of response and an equivalent median PFS and OS when compared with patients presenting with low LDH levels. These findings may be considered in accordance with the observations derived from the CONFIRM-1 and -2, thus confirming an improved clinical outcome for patients with high LDH levels when treated with an anti-VEGF therapy. However, in contrast to the PTK/ZK results, we evidenced an improved response rate in the high-LDH group treated with bevacizumab (which is not present in neither the Hecht nor the Van Cutsem study; Hecht et al, 2011; Van Cutsem et al, 2011), whereas no advantages could be seen in our series with regard to median PFS. Many factors may have contributed to these apparently different profiles of activity. The different antiangiogenic treatment used (an anti-VEGF monoclonal antibody in our case $v s$ an oral VEGF receptor tyrosine kinase inhibitor in the CONFIRM trials) should be considered relevant. Although we have limited data on the activity profile of PTK/ZK, we can in fact assume that vatalanib may be more effective in improving PFS than response rate in the high-LDH subgroup of patients, whereas bevacizumab demonstrated also an impact in determining response rate across trials. We also know that radiological evaluation of response could represent a confounding factor for patients receiving antiangiogenic treatment (Chun et al, 2009).

In our experience, treatment with bevacizumab seemed to be capable of improving clinical outcome in a specific group of patients who usually present with an adverse natural history. In this group of patients, bevacizumab seemed to act as a clinical outcome 'equaliser' inducing a reversal of a poor prognosis. The finding of an improved response rate and median PFS for patients with high pre-treatment LDH levels receiving bevacizumab over patients with the same LDH levels not receiving bevacizumab could represent a corroboration to this observation.

The interaction test between LDH levels and treatment effect, in the bevacizumab group and historical control group, in fact suggested that the improved outcome in high-LDH patients was significantly associated with the effect of bevacizumab on PFS. Our results, if confirmed in a larger data set, may have relevant implications for the choice of a first-line treatment for advanced colorectal cancer patients. We can in fact speculate that, in colorectal cancer patients with high LDH levels, the use of an antiangiogenic treatment in combination with chemotherapy may improve clinical outcome and allow a better management of the metastatic disease.

\section{REFERENCES}

Azuma M, Shi M, Danenberg KD, Gadner H, Barrett C, Jacques CJ, Sherod A, Igbal S, El-Khoueiry A, Yand D, Zhang W (2007) Serum lactate dehydrogenase levels and glycolysis significantly correlate with tumor VEGFA and VEGFR expression in metastatic CRC patients. Pharmacogenomics 8: $1705-1713$

Brizel DM, Schroeder T, Scher RL, Walenta S, Clough RW, Dewhirst MW, Mueller-Klieser W (2001) Elevated tumor lactate concentrations predict for an increased risk of metastases in head-and-neck cancer. Int J Radiat Oncol Biol Phys 51: 349-353

Chun YS, Vauthey JN, Boonsirikamchai P (2009) Association of computed tomography morphologic criteria with pathologic response and survival in patients treated with bevacizumab for colorectal liver metastases. JAMA 2: $2338-2344$

De Gramont A, Figer A, Seymour M, Homerin M, Hmissi A, Cassidy J, Boni C, Cortes-Funes H, Cervantes A, Freyer G, Papamichael D, Le Bail N, Louvet C, Hendler D, de Braud F, Wilson C, Morvan F, Bonetti A (2000) Leucovorin and fluorouracil with or without oxaliplatin as first-line treatment in advanced colorectal cancer. J Clin Oncol 18: $2938-2947$

Douillard JY, Cunningham D, Roth AD, Navarro M, James RD, Karasek P, Jandik P, Iveson T, Carmichael J, Alakl M, Gruia G, Awad L, Rougier P (2000) Irinotecan combined with fluorouracil compared with fluorouracil alone as first-line treatment for metastatic colorectal cancer: a multicentre randomized trial. Lancet 355: $1041-1047$

Folkman J (1971) Tumor angiogenesis: therapeutic implications. N Engl $J$ Med 285: $1182-1186$

Harris AL (2002) Hypoxia - a key regulatory factor in tumour growth. Nat Rev Cancer 2: 38-47

Hecht JR, Trarbach T, Hainsworth JD, Major P, Jager E, Wolff RA, LloydSalvant K, Bodoky G, Pendergrass K, Berg W, Chen BL, Jalava T, Meinhardt G, Laurent D, Lebwohl D, Kerr D (2011) Randomized, placebo-controlled, phase III study of first-line oxaliplatin-based chemotherapy plus PTK787/
ZK222584, an oral vascular endothelial growth factor receptor inhibitor, in patients with metastatic colorectal adenocarcinoma. J Clin Oncol 20: $1997-2003$

Hurwitz H, Fehrenbacher L, Novotny W, Cartwright T, Hainsworth J, Heim W, Berlin J, Baron A, Griffing S, Holmgren E, Ferrara N, Fyfe G, Rogers B, Ross R, Kabbinavar F (2004) Bevacizumab plus irinotecan, fluorouracil, and leucovorin for metastatic colorectal cancer. $N$ Engl J Med 350: 2335-2342

Koehne CH, Van Cutsem E, Wils J, Bokemeyer C, El-Serafi M, Lutz MP, Lorenz M, Reichardt P, Ruckle-Lanz H, Frickhofen N, Fuchs R, Mergenthaler HG, Langenbuch T, Vanhoefer U, Rougier P, Voigtmann R, Muller L, Genicot B, Anak O, Nordlinger B (2005) Phase III study of weekly high-dose infusional fluorouracil plus folinic acid with or without irinotecan in patients with metastatic colorectal cancer: European Organisation for Research and Treatment of Cancer Gastrointestinal Group Study 40986. J Clin Oncol 23: 4856-4865

Koukourakis MI, Giatromanolaki A, Simopoulos C, Polychronidis A, Sivridis E (2005) Lactate dehydrogenase 5 (LDH5) relates to up-regulated hypoxia inducible factor pathway and metastasis in colorectal cancer. Clin Exp Metast 22: 25-30

Koukourakis MI, Giatromanolaki A, Sivridis E (2003) Lactate dehydrogenase isoenzymes 1 and 5: differential expression by neoplastic and stromal cells in non-small cell lung cancer and other epithelial malignant tumors. Tumour Biol 24: 199-202

Koukourakis MI, Giatromanolaki A, Sivridis E, Gatter KC, Harris AL (2006) Lactate dehydrogenase 5 expression in operable colorectal cancer: strong association with survival and activated vascular endothelial growth factor pathway - a report of the Tumour Angiogenesis Research Group. J Clin Oncol 24: $4301-4308$

Koukourakis MI, Giatromanolaki A, Sivridis E, Gatter KC, Trarbach T, Folprecht G (2011) Prognostic and predictive role of lactate dehydrogenase 5 expression in colorectal cancer patients treated with PTK787/ZK 222584 (vatalanib) antiangiogenic therapy. Clin Cancer Res 15: $4892-4900$ 
Maxwell PH, Pugh CW, Ratcliffe PJ (2001) Activation of the HIF pathway in cancer. Curr Opin Genet Dev 11: 293-299

Tas F, Aydiner A, Demir C, Topuz E (2001b) Lactate dehydrogenase levels at presentation predict outcome of patients with limited stage small-cell lung cancer. Am J Clin Oncol 24: 376-378

Tas F, Aykan F, Alici S, Kaytan E, Aydiner A, Topuz E (2001a) Prognostic factors in pancreatic carcinoma: serum LDH levels predict survival in metastatic disease. Am J Clin Oncol 24: 547-550

Tournigand C, André T, Achille E, Lledo G, Flesh M, Mery-Mignard D, Quinaux E, Couteau C, Buyse M, Ganem G, Landi B, Colin P, Louvet C, de Gramont A (2004) FOLFIRI followed by FOLFOX6 or the reverse sequence in advanced colorectal cancer: a randomized GERCOR study. J Clin Oncol 22: 229-237

Tournigand C, Cervantes A, Figer A, Lledo G, Flesch M, Buyse M, Mineur L, Carola E, Etienne PL, Rivera F, Chirivella I, Perez-Staub N, Louvet C (2011)
Randomized, placebo-controlled, phase III study of oxaliplatin, fluorouracil and leucovorin with or without PTK787/ZK222584 in patients with previously treated metastatic colorectal adenocarcinoma. J Clin Oncol 20: 2004-2010

Van Cutsem E, Bajetta E, Valle J, Köhne CH, Hecht JR, Moore M, Germond C, Berg W, Chen BL, Jalava T, Lebwohl D, Meinhardt G, Laurent D, Lin E (2011) Randomized, placebo-controlled, phase III study of oxaliplatin, fluorouracil, and leucovorin with or without PTK787/ZK 222584 in patients with previously treated metastatic colorectal adenocarcinoma. J Clin Oncol 29: 2004-2010

Wu XZ, Ma F, Wang XL (2010) Serological diagnostic factors for liver metastasis in patients with colorectal cancer. World J Gastroenterol 16: $4084-4088$

Yuce K, Baykal C, Genc C, Al A, Ayhan A (2001) Diagnostic and prognostic value of serum and peritoneal fluid lactate dehydrogenase in epithelial ovarian cancer. Eur J Gynaecol Oncol 22: 228-232

This work is published under the standard license to publish agreement. After 12 months the work will become freely available and the license terms will switch to a Creative Commons Attribution-NonCommercial-Share Alike 3.0 Unported License. 